\title{
A Model for Soft Interactions based on the CGC/Saturation Approach and the BFKL Pomeron
}

\author{
Errol Gotsman ${ }^{1, a}$ \\ ${ }^{1}$ School of Physics and Astronomy, Tel Aviv University, Ramat Aviv, Israel
}

\begin{abstract}
We propose a consistent approach to describe soft interactions at LHC and higher energies based on the BFKL Pomeron and CGC/saturation.We assume that the same Pomeron describes both "soft" and "hard" interactions. Having eight parameters we obtain a good description of the elastic and inclusive proton-proton data at energies $\mathrm{W} \leq 0.546 \mathrm{TeV}$.
\end{abstract}

\section{Introduction}

Strong interactions at high energy are considered a typical example of processes that occur at long distances, where the unknown mechanism which confines quarks and gluons, plays a confusing role in undermining all efforts to treat these process theoretically. Consequently, the description of these soft processes are usually made using high energy phenomenology, based on Pomeron-Reggeon calculus [1].

To ameliorate this difficulty, the Tel Aviv group (Gotsman, Levin and Maor) has suggested and formalized an approach, which attempts to describe soft interactions by adopting well established aspects of QCD in the saturation region [2], and extending these to the region of soft interactions (low $Q^{2}$ ). Our basic assumption, is that the BFKL Pomeron [3], at high energies, describes both hard and soft scattering.

The processes that our model has been successfully applied are proton-proton scattering over the energy range $546 \mathrm{GeV} \leq \mathrm{W} \leq 57 \mathrm{TeV}$ : include total, elastic and diffractive cross sections as well as the forward slope [4]: inclusive production [5]:long range correlations [6]: and the survival probability of central exclusive production [7].

To illustrate our approach, I will limit myself and only discuss our results, and compare these to the relevent experimental data for the proton-proton total, elastic and differential cross sections, as well as inclusive production.

\section{Background}

1. In the Regge limit of pQCD, when $s \gg \Lambda_{\text {hard }}$, as the energy increases the parton density becomes more dense, and the scattering amplitude $\mathrm{A}(\mathrm{s}, \mathrm{t})$ grows.

2. As long as densities are not too high, growth is described by BFKL evolution equation.

\footnotetext{
ae-mail: gotsman@post.tau.ac.il
} 
3. Density becomes higher as $\mathrm{A}(\mathrm{s}, \mathrm{t}) \rightarrow 1$, and one enters a regime called "saturation", where the BFKL evolution fails.

4. Non linearities lead to "saturation" + "unitarization" of $\mathrm{A}(\mathrm{s}, \mathrm{t})$.

5. Balitsky-Kovchegov equation [8] is the simplest and most accurate way to describe the saturation regime of QCD. It is non-linear and resums QCD fan diagrams in the LLA.

\section{Phenomenological Input}

One of the most well established principles of high energy scattering is that the resulting cross section should not violate the Froissart bound (F.B.).

There is a very simple criterion due to Heisenberg (quoted by Kovner and Wiedemann [9]) relating the impact parameter dependence of the profile of the matter density in any target, and the violation of the F.B.

(i) If the matter density decays exponentially at the periphery i.e. $\rho(b) \propto e^{-m b}$, then for the production of the lightest particle of mass $\mathrm{m},\left(E \sim \frac{s}{m}\right)$, the target energy density is $E \rho(b)$. Scattering can only take place for $b \leq b_{\max }$. Since

$$
E e^{-m b_{\max }}=m \Rightarrow b_{\max } \leq \frac{1}{m} \ln \left(\frac{s}{m^{2}}\right) \Rightarrow \sigma_{t o t} \leq b_{\max }^{2} \leq \frac{c}{m^{2}} \ln ^{2}\left(\frac{s}{m^{2}}\right)
$$

this satisfies the F.B.

(ii) Conversely, if the density distribution in the target is power-like, i.e. $\rho(b) \propto b^{-\lambda}$. Hence,

$$
E e^{m} b_{\max }=m \Rightarrow b_{\max }^{-\lambda} \sim\left(\frac{s}{m^{2}}\right)^{1 / 2} \Rightarrow \sigma_{t o t} \leq b_{\max }^{2} \sim\left(\frac{s}{m^{2}}\right)^{\frac{2}{\lambda}} .
$$

i.e. $\sigma_{t o t} \propto$ a power of $\mathrm{s}$, which leads to a violation of the F.B.

In building our model, we need to overcome a deficiency i.e. the fact that the BFKL Pomeron does not lead to shrinkage of the diffractive peak, and has no slope for the Pomeron trajectory. This can be cured by introducing a non-perturbative correction at large impact parameter, which also assures satisfying the Froissart-Martin bound for $\sigma_{t o t}$.

Consequently, in our model we fix the large $\mathrm{b}$ behaviour by assuming that the saturation momentum $Q_{s}^{2}$, has the following form:

$Q_{s}^{2}(b, Y)=Q_{0 s}^{2}\left(b, Y_{0}\right) e^{\lambda\left(Y-Y_{0}\right)}$ and $Q_{0 s}^{2}\left(b, Y_{0}\right)=\left(m^{2}\right)^{\left(1-\frac{1}{\bar{\gamma}}\right)}[S(b, m)]^{\frac{1}{\bar{\gamma}}} ; S(b, m)=\frac{m^{2}}{2 \pi} e^{-m b}$ and $\bar{\gamma}=$ $0.63=1-\gamma_{c r}$.

The parameter $\lambda=\bar{\alpha}_{S} \chi\left(\gamma_{c r}\right) /\left(1-\gamma_{c r}\right)$, in leading order of perturbative QCD $(\lambda=0.2$ to 0.3$)$.

The parameter $\mathrm{m}$ which is introduced to describe the large $\mathrm{b}$ behaviour, and determines the typical sizes of dipoles inside the hadron.

Our model also includes two additional scales $m_{1}$ and $m_{2}$, which describe two typical sizes in the proton wave function. One can associated these with: (i) the distance between the constituent quarks; size of the proton $R_{p} \approx \frac{1}{m_{1}}$. and (ii) $m_{2}$ can be associated with the size of the constituent quark; $R_{q} \approx \frac{1}{m_{2}}$.

Altinoluk et al [10] have proved the equivalence of the CGC/saturation approach and the BFKL Pomeron calculus for a wide range of rapidities, $Y \leq \frac{2}{\Delta_{\mathrm{BFKL}}} \ln \left(\frac{1}{\Delta_{\mathrm{BFKL}}^{2}}\right)$. 


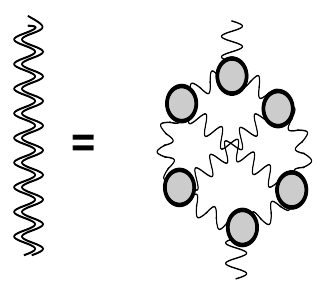

a)

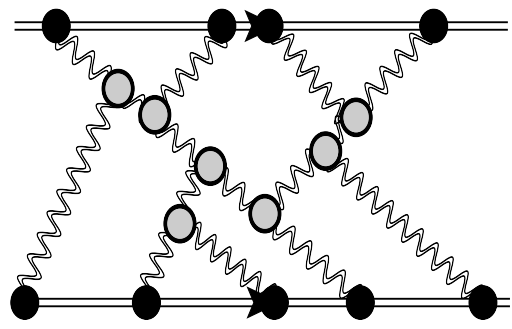

b)

Figure 1. a) Dressed Pomeron in MPSI approximation and b) Sum of net diagrams. Wavy lines describe BFKL Pomerons. The grey blobs stand for triple Pomeron vertices, while black blobs show the hadron-Pomeron vertex $\mathrm{g}(\mathrm{b})$.

\subsection{Dressed Pomeron in MPSI approximation}

For a description of the process see Fig.1.

Since the typical rapidity is $O\left(Y-Y_{i}\right) \approx \frac{1}{\triangle_{B F K L}}$, only large Pomeron loops with rapidity $O(Y)$ contribute at high energies $\rightarrow$ can sum such loops using MPSI approximation.

For the BFKL Pomeron $\lambda=4.88 \bar{\alpha}_{s}$ while $\Delta_{B F K L}=4 \ln 2 \bar{\alpha}_{s} \approx 0.2$.

The resulting Green function of the Dressed Pomeron is given by:

$$
\begin{aligned}
& G_{\boldsymbol{P}}^{\text {dressed }}\left(Y-Y_{0}, r, R, b\right)= \\
& a^{2}\left\{1-\exp \left(-T\left(Y-Y_{0}, r, R, b\right)\right)\right\}+2 a(1-a) \frac{T\left(Y-Y_{0}, r, R, b\right)}{1+T\left(Y-Y_{0}, r, R, b\right)} \\
& +(1-a)^{2}\left\{1-\exp \left(\frac{1}{T\left(Y-Y_{0}, r, R, b\right)}\right) \frac{1}{T\left(Y-Y_{0}, r, R, b\right)} \Gamma\left(0, \frac{1}{T\left(Y-Y_{0}, r, R, b\right)}\right)\right\}
\end{aligned}
$$

The BFKL amplitude in the vicinity of the saturation scale is denoted by

$$
T\left(Y-Y_{0}, r, R, b\right)=\frac{\bar{\alpha}_{S}^{2}}{4 \pi} G_{P}(z \rightarrow 0)=\phi_{0}\left(r^{2} Q_{s}^{2}(R, Y, b)\right)^{1-\gamma_{c r}}=\phi_{0} S(b) e^{\lambda\left(1-\gamma_{c r}\right) Y}
$$

$G_{P}(Y, r, R)$ denotes the BFKL Pomeron's Green function.

$\mathrm{z}=\ln \left(r^{2} Q_{s}^{2}(b, Y)\right), \mathrm{a}=0.65$, the critical anamolous dimension is given by $\gamma_{c r} \approx 0.37$.

\subsection{Parameters of the Model}

We used two inputs: $r=R$ and $Q_{s}^{2}=\left(1 /\left(m^{2} R^{2}\right)\right) S(b) \exp (\lambda Y)$.

The phenomenological profile function $S(b)=\frac{m^{2}}{2 \pi} e^{-m b}$ with normalization $\int d^{2} b S(b)=1$. We need to introduce four constants: $g_{i}$ and $m_{i}(i=1,2)$, to describe the vertices of the hadronPomeron interaction

$$
g_{i}(b)=g_{i} S_{\boldsymbol{P}}(b) \text { with } S_{\boldsymbol{P}}(b)=\frac{m_{i}^{3} b}{4 \pi} K_{1}\left(m_{i} b\right) ; S_{\boldsymbol{P}}(b) \stackrel{\text { Fourier image }}{\longrightarrow}\left(\frac{m_{i}^{2}}{q^{2}+m_{i}^{2}}\right)^{2}
$$


The opacity is given by:

$$
\Omega_{i, k}(Y ; b)=\int d^{2} b^{\prime} \frac{g_{i}\left(\vec{b}^{\prime}\right) g_{k}\left(\vec{b}-\vec{b}^{\prime}\right) \bar{G}_{\boldsymbol{P}}^{\text {dressed }}(Y)}{1+1.29 \bar{G}_{\boldsymbol{P}}^{\operatorname{dressed}}(Y)\left[g_{i}\left(\vec{b}^{\prime}\right)+g_{k}\left(\vec{b}-\vec{b}^{\prime}\right)\right]},
$$

where $\bar{G}_{\boldsymbol{P}}^{\text {dressed }}(Y)=\int d^{2} b^{\prime \prime} G_{\boldsymbol{P}}^{\text {dressed }}\left(Y ; b^{\prime \prime}\right)$.

The factor 1.29 originates from estimates of the $3 \boldsymbol{P}$ vertex in the CGC/saturation approach.

\subsection{Basic formalism for the two channel model}

Following Good-Walker [11] the observed physical hadronic and diffractive states are written

$$
\psi_{h}=\alpha \Psi_{1}+\beta \Psi_{2} ; \quad \psi_{D}=-\beta \Psi_{1}+\alpha \Psi_{2} ; \quad \text { where } \quad \alpha^{2}+\beta^{2}=1
$$

Functions $\psi_{1}$ and $\psi_{2}$ form a complete set of orthogonal functions $\left\{\psi_{i}\right\}$ which diagonalize the interaction matrix $\mathbf{T}$

$$
A_{i, k}^{i^{\prime} k^{\prime}}=<\psi_{i} \psi_{k}|\mathbf{T}| \psi_{i^{\prime}} \psi_{k^{\prime}}>=A_{i, k} \delta_{i, i^{\prime}} \delta_{k, k^{\prime}}
$$

The unitarity constraints can be written as

$$
2 \operatorname{Im} A_{i, k}(s, b)=\left|A_{i, k}(s, b)\right|^{2}+G_{i, k}^{i n}(s, b)
$$

At high energies a simple solution to this equation is

$$
\begin{gathered}
A_{i, k}(s, b)=i\left(1-\exp \left(-\frac{\Omega_{i, k}(s, b)}{2}\right)\right) \\
G_{i, k}^{i n}(s, b)=1-\exp \left(-\Omega_{i, k}(s, b)\right) .
\end{gathered}
$$

$G_{i, k}^{i n}(s, b)$ denotes the contribution of all non-diffractive inelastic processes.

\subsection{Physical observables for elastic and low mass diffraction}

elastic amplitude:

elastic observables :

$$
\begin{aligned}
& a_{e l}(s,)=i\left(\alpha^{4} A_{1,1}+2 \alpha^{2} \beta^{2} A_{1,2}+\beta^{4} A_{2,2}\right) ; \\
& \sigma_{t o t}=2 \int d^{2} b a_{e l}(s, b) ; \quad \sigma_{e l}=\int d^{2} b\left|a_{e l}(s, b)\right|^{2} ;
\end{aligned}
$$

optical theorem :

single diffraction:

$$
2 \operatorname{Im} A_{i, k}(s, t=0)=2 \int d^{2} b \operatorname{Im} A_{i, k}(s, b)=\sigma_{e l}+\sigma_{i n}=\sigma_{t o t} ;
$$

double diffraction :

$$
\sigma_{s d}^{G W}=\int d^{2} b\left(\alpha \beta\left\{-\alpha^{2} A_{1,1}+\left(\alpha^{2}-\beta^{2}\right) A_{1,2}+\beta^{2} A_{2,2}\right\}\right)^{2} ;
$$

$$
\sigma_{d d}^{G W}=\int d^{2} b \alpha^{4} \beta^{4}\left\{A_{1,1}-2 A_{1,2}+A_{2,2}\right\}^{2} .
$$

'GW' denotes the Good -Walker component, that is responsible for diffraction in the small mass region.

The parameters of our best fit are given in Table 1, while our results and comparison with the relevant experimental data are displayed in Fig.2. 

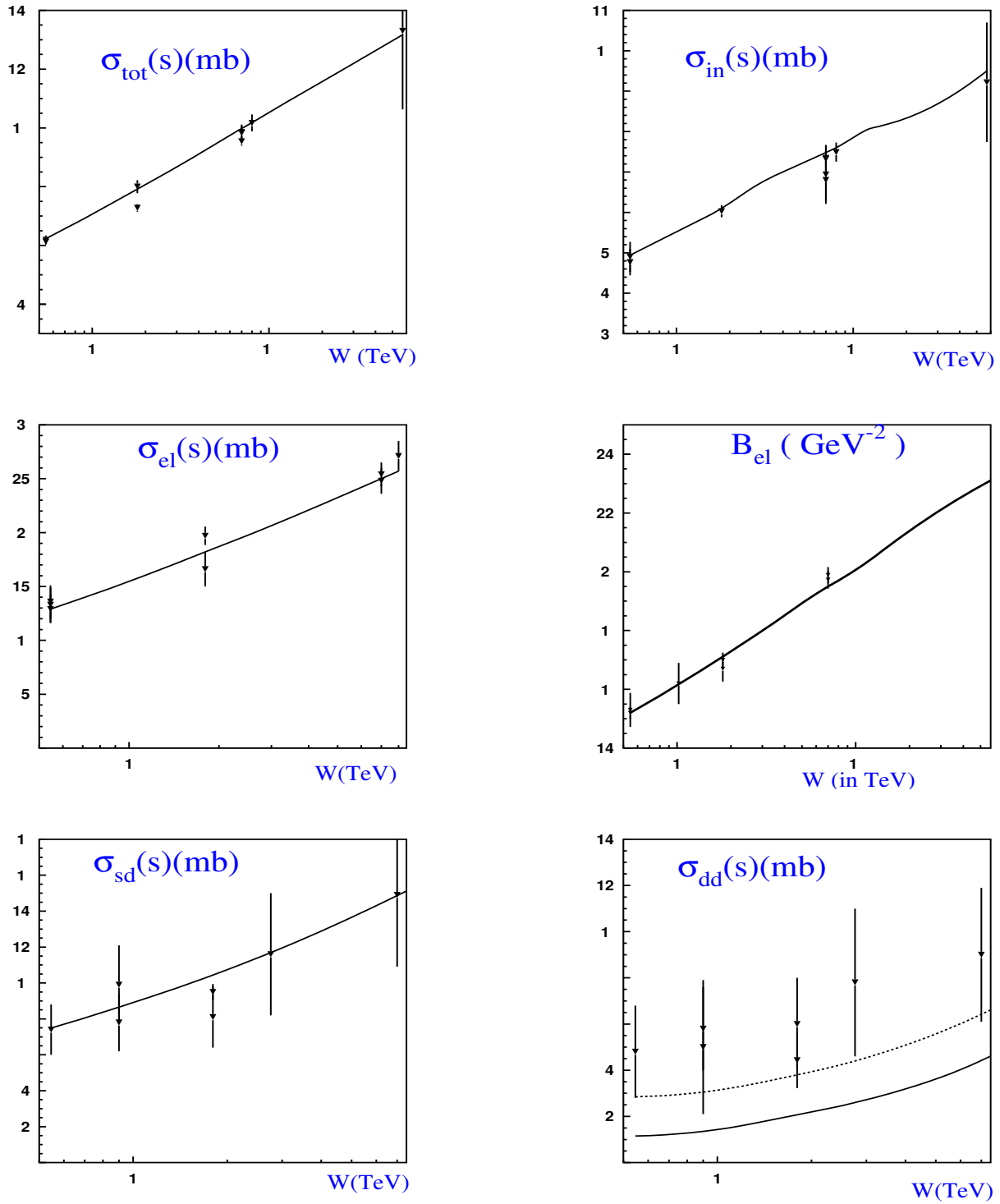

Figure 2. Comparison of model results with experimental data: the energy behaviour of the total (Fig.2-a), inelastic (Fig.2-b), elastic cross section (Fig.2-c), as well as the elastic slope $B_{e l}$ (Fig.2-d), and single diffraction (Fig.2-e) and double diffraction (Fig.2-f) cross sections. The solid lines show our fit. The data has been taken from Ref.[12] for energies less than the LHC energy. At the LHC energy for total and elastic cross sections we use [13] data and for the single and double diffraction cross sections are taken from ref.[14]. The dotted line in Fig.2-f) which is obtained assuming factorization is discussed in [4]. 
Table 1. Table 1. Fitted parameters of the model

\begin{tabular}{|l|l|l|l|l|l|l|l|l|}
\hline model & $\lambda$ & $\phi_{0}$ & $g_{1}\left(\mathrm{GeV}^{-1}\right)$ & $g_{2}\left(\mathrm{GeV}^{-1}\right)$ & $m(\mathrm{GeV})$ & $m_{1}(\mathrm{GeV})$ & $m_{2}(\mathrm{GeV})$ & $\beta$ \\
\hline 2 channel & 0.38 & 0.0019 & 110.2 & 11.2 & 5.25 & 0.92 & 1.9 & 0.58 \\
\hline
\end{tabular}

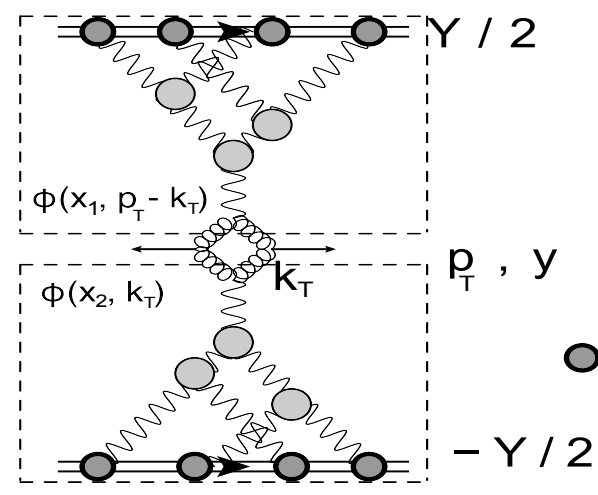

$\mathrm{h}$ a)
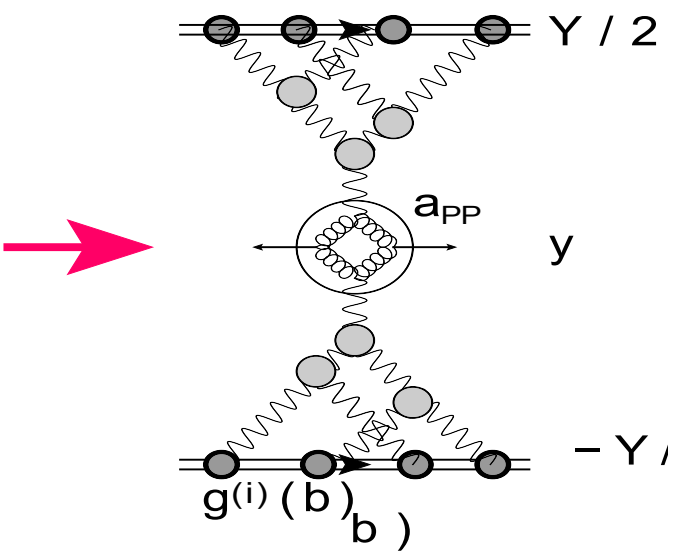

Figure 3. The graphical representation of Eqn. (1) (Fig-3a). Wavy lines denote the BFKL Pomerons, while the helical lines illustrate the gluons. In Fig-3b the Mueller diagram for inclusive production is shown.

\section{Application of the CGC/saturation approach to Inclusive Production}

See [5] for details.

Inclusive production occurs in two stages:

First stage: Production of a mini-jet with typical transverse momentum $Q_{s}: Q_{s}$ (saturation scale)» soft scale.

Second stage: Decay of minijets into hadrons, which is treated phenomenologically.

For mini-jet production we use the $k_{T}$ factorization formula:

$$
\frac{d \sigma}{d y d^{2} p_{T}}=\frac{2 \pi \bar{\alpha}_{S}}{p_{T}^{2}} \int d^{2} k_{T} \phi_{G}^{h_{1}}\left(x_{1} ; \vec{k}_{T}\right) \phi_{G}^{h_{2}}\left(x_{2} ; \vec{p}_{T}-\vec{k}_{T}\right)
$$

where $\phi_{G}^{h_{i}}$ denotes the probability to find a gluon that carries the fraction $x_{i}$ of energy with $k_{\perp}$ transverse momentum. and $\bar{\alpha}_{S}=\alpha_{s} N_{c} / \pi$, with the number of colours equal to $N_{c}$.

Where $\frac{1}{2} Y+y=\ln \left(1 / x_{1}\right)$ and $\frac{1}{2} Y-y=\ln \left(1 / x_{2}\right) . \phi_{G}^{h_{i}}$ is the solution of the Balitsky-Kovchegov(BK) non-linear evolution equation, and can be viewed as the sum of 'fan' diagrams of the BFKL Pomeron interactions, shown in Fig-3.

For the sake of simplicity all other indices in $\phi\left(x_{1}, p_{T}-k_{T}\right)$ and $\phi\left(x_{2}, k_{T}\right)$ are omitted. 
Eqn.(1) can be rewritten as a Mueller diagram ( Fig-3b), and the inclusive cross section is given by:

$$
\begin{gathered}
\frac{d \sigma}{d y}=\int d^{2} p_{T} \frac{d \sigma}{d y d^{2} p_{T}}=a_{\boldsymbol{P P}} \ln \left(W / W_{0}\right)\left\{\alpha^{4} \operatorname{In}^{(1)}\left(\frac{1}{2} Y+y\right) \operatorname{In}^{(1)}\left(\frac{1}{2} Y-y\right)\right. \\
+\alpha^{2} \beta^{2}\left(\operatorname{In}^{(1)}\left(\frac{1}{2} Y+y\right) \operatorname{In}^{(2)}\left(\frac{1}{2} Y-y\right)+\operatorname{In}^{(2)}\left(\frac{1}{2} Y+y\right) \operatorname{In}^{(1)}\left(\frac{1}{2} Y-y\right)\right) \\
\left.+\beta^{4} \operatorname{In}^{(2)}\left(\frac{1}{2} Y+y\right) \operatorname{In}^{(2)}\left(\frac{1}{2} Y-y\right)\right\} \\
\operatorname{In}^{(i)}(y)=\int d^{2} b N^{B K}\left(g^{(i)} S\left(m_{i}, b\right) \tilde{G}_{\boldsymbol{P}}(y)\right)
\end{gathered}
$$

where $\tilde{G}_{\boldsymbol{P}}(y)=\phi_{0} \exp \left(\lambda\left(1-\gamma_{c r}\right) y\right)$

and $N^{B K}$ is an approximation to the numerical solution of the BK equation.

The mass of mini jet is given by $m_{\text {jet }}^{2}=2 m_{\text {soft }} p_{T}$.

Since the typical transverse momentum is equal to the saturation scale, we have

$$
\frac{m_{j e t}^{2}}{p_{T}^{2}}=\frac{2 m_{\text {soft }}}{Q_{s}(W)}=r_{0}^{2}\left(\frac{W}{W_{0}}\right)^{-\frac{1}{2} \lambda}
$$

Values of parameters have been extracted from the diffractive and elastic data.

The only free parameters are $a_{\boldsymbol{P P}}$ and $r_{0}^{2}$.

Our curves are calculated for $a_{\boldsymbol{P P}}=0.21$ and $r_{0}^{2}=8$, which have been determined from the experimental data.

\subsection{Our Model Results for Inclusive Production}

The results of our fit and the relevent experimental data are displayed in Fig (4) and Fig.(5).

\section{Conclusions}

Our constructed model based on the BFKL Pomeron and the CGC/saturation approach, successfully describes data in the Regge region, for high energy hadron scattering.

We do not require that the soft Pomeron appear as a Regge pole.

We suggest a procedure where the matching with long distance physics (where confinement of quarks and gluons is essential) can be reached within the CGC/saturation approach.

We have also shown that our formalism for soft (long distance) interactions, successfully describes inclusive production.

\section{References}

[1] P.D.B. Collins, An introduction to Regge theory and high energy physics(Cambridge University Press, 1977) 


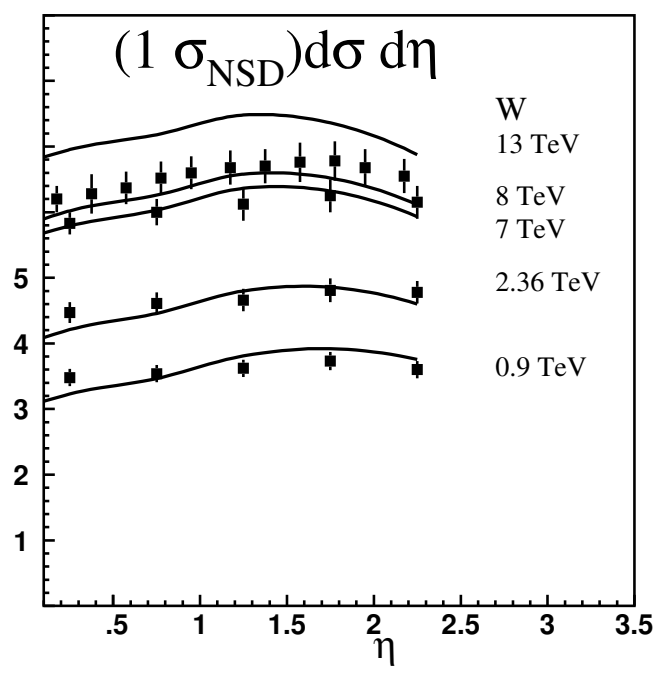

(a)

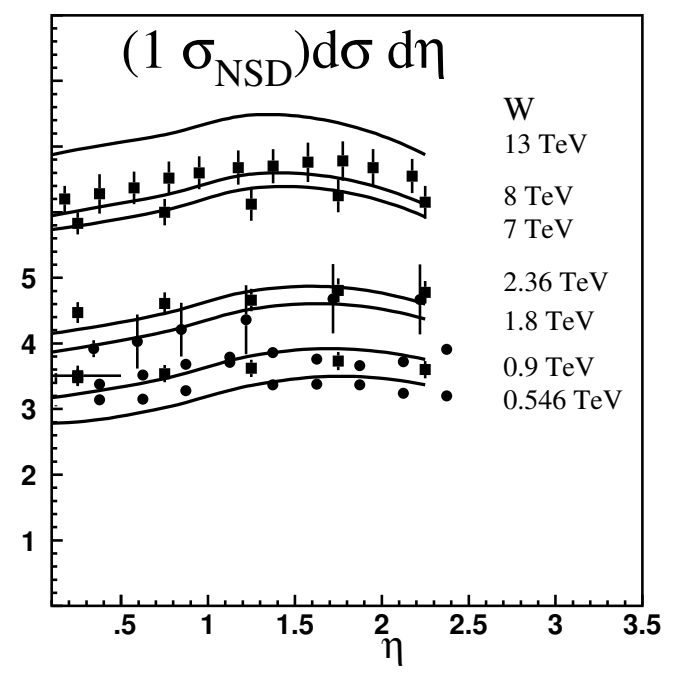

(b)

Figure 4. The single inclusive density $\left.\left(1 / \sigma_{N S D}\right) d \sigma / d \eta\right)$ versus energy. The data were taken from ALICE [18],CMS [19], ATLAS [22] and from PDG [17]. The description of the CMS data is plotted in in fig.(2-a) while fig.(2-b) presents the comparison with all inclusive spectra with $\mathrm{W} \geq 0.546 \mathrm{TeV}$.

[2] Yuri V. Kovchegov and Eugene Levin, Quantum Chromodynamics at High Energies(Cambridge Monographs on Particle Physics, Nuclear Physics and Cosmology, Cambridge University Press, 2012).

[3] E.A. Kuraev, L. N. Lipatov and F. S. Fadin, Sov.Phys.JETP 45, 199 (1977); Ya. Ya. Balitsky and L. N. Lipatov, Sov.J.Nucl.Phys. 28, 22 (1978).

[4] E. Gotsman, E. Levin and U. Maor, Eur.Phys.J. C75, 179 (2015).

[5] E. Gotsman, E. Levin and U. Maor, Phys.Lett. B746, 154 (2015).

[6] E. Gotsman, E. Levin and U. Maor, Eur.Phys.J. C75, 518 (2015).

[7] E. Gotsman, E. Levin and U. Maor, Eur.Phys.J. C76, 177 (2016).

[8] I. Balitsky, Phys.Rev. D60 014020 (1999); Y.V. Kovchegov, Phys. Rev D60, 034008 (1999).

[9] A.Kovner and U. Wiedemann, Phys. Letts. B551 311 (2003).

[10] T. Altinoluk et al JHEP, 1404, 075 (2014).

[11] M.L. Good and W.D. Walker, Phys.Rev. 120, 1857 (1960).

[12] J. Beringer et al [particle Data Group], Phys. Rev. D86, 010001 (2012).

[13] F. Ferro [Totem collaboration] AIP Conf.Proc. 1350, 172 (2011); G.Antchev et. al. [TOTEM collaboration], Europhys. Lett.96, 21002 (2011),Europhys. Lett. 95, 41001 (2011).Nucl.Phys. 899, 527 (2015).

[14] M. G. Poghosyan, J.Phys.G38, 124044 (2011), [ALICE Collaboration], arXiv:0911.5430[hepex].

[15] G. Aad et al. [ATLAS Collaboration], Nature Commun. 2, 463 (2011); Phys. Lettrs. B754, 214 (2016). 


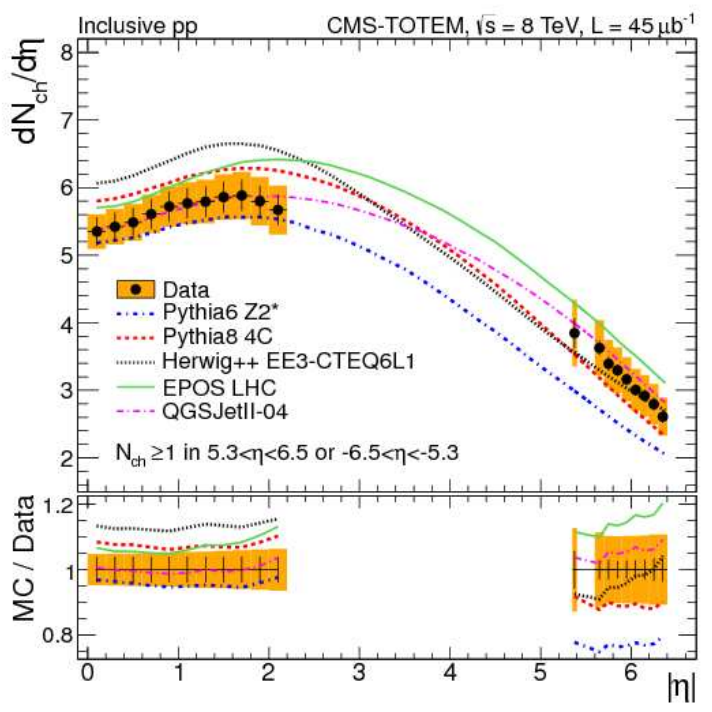

(a)

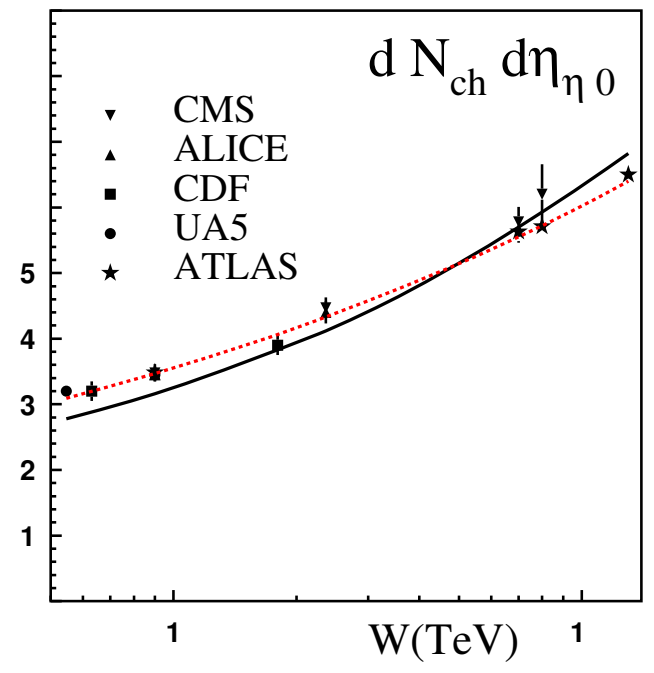

(b)

Figure 5. The comparison of the inclusive production at $W=8 \mathrm{TeV}$ with the Monte Carlo models is shown in fig (a). The figure is taken from [CMSIN and TOTEMIN] In fig.(b) $d N_{c h} / d \eta$ at $\eta=0$ versus energy $W$ is displayed. Our estimates are shown by the solid line. The dotted line corresponds to fit: $0.725\left(W / W_{0}\right)^{0.23}$ with $W_{0}=1 G e V$. The data are taken from [17],[18],[19],[20],[21],[22].

[16] CMS Collaboration,Phys. Letts. B749, 242, (2015).

[17] J. Beringer, et al. (Particle Data Group), Phys.Rev. D86,010001 (2012).

[18] K. Aamodt et al.[ALICE Collaboration], Eur. Phys. J.C68, 89 (2010); [arXiv:1004.3034 [hepex]]; ALICE Collaboration, Eur. Phys. J.C65, 65 (2010) 111 [arXiv:0911.5430 [hep-ex]].

[19] S. Chatrchyan et al. [CMS and TOTEM Collaborations], Eur. Phys. J. C74, 3053 (2014), [arXiv:1405.0722 [hep-ex]]; V. Khachatryan et al. [CMS Collaboration], Phys. Rev. Lett. 105, 022002 (2010); [arXiv:1005.3299 [hep-ex]]. V. Khachatryan et al.[CMS Collaboration], JHEP 1002, 041 (2010) [arXiv:1002.0621 [hep-ex].

[20] T. A. Altonen, et al [CDF Collaboration] Phys. Rev. D79, 112005 (2009).

[21] G.J. Alner, et al [UA5 Collaboration] Z.Phys. C33 , 1 (1986).

[22] [ATLAS Collaboration] arXiv:1003.3124 [hep-ex]. 